Tomasz Kaczmarek

Università di Łódź

Istituto di Romanistica

Dipartimento di Letterature Romanze

https://doi.org/10.18778/8220-478-0.15

\title{
PIRANDELLO A ŁÓDŹ \\ OVVERO ADAM HANUSZKIEWICZ METTE IN SCENA COSİ È (SE VI PARE)
}

\begin{abstract}
Nel presente articolo si tratta dell'allestimento di Così è (se vi pare) di Pirandello che ebbe un successo notevole sul palcoscenico del Teatr Powszechny a Łódź nel 1989. Questa rappresentazione fu importante non solo per la carriera del regista, Adam Hanuszkiewicz, ma anche per la ricezione della produzione pirandelliana di cui le vicende non furono fino ad allora particolarmente favorevoli. Essa propose nuove possibilità interpretative fin qui celate dell'opera dell'autore italiano. Di fatti, oltre a trasgredire le convenzioni sceniche, secondo alcuni ormai desuete, il Siciliano mise avanti una nuova drammaturgia frammentaria e apparentemente incompiuta che si concentra piuttosto sulla "vita" che sull'"azione prettamente agonistica". Esponendo un nuovo paradigma del "dramma-della-vita" che sta in netta oposizione al "dramma-nella-vita", ossia "dramma assoluto", egli diede vita alla drammaturgia moderna e contemporanea. Aderendo all'ottica teatrale e drammatica di Pirandello, Hanuszkiewicz tende dunque a liberare l'autore di Enrico IV dalle tradizionali interpretazioni che si focalizzavano essenzialmente sul suo sistema chiamato "pirandellismo" oppure sul presunto "esistenzialismo". Il regista polacco non esitò ad adottare il dramma ai tempi moderni, senza però intaccare i presupposti "ideologici" dell'opera: Hanuszkiewicz la rilesse secondo la chiave contemporanea ed anche profondamente personale, scoperchiandone il suo carattere sempre attuale nonché universale che poteva essere accolto con comprensione e ammirazione non solamente in Italia. Larticolo ripercorre quindi la fortuna dello spettacolo che, al pari dei Giganti della montagna, messo in scena da Izabella Cywińska (1974), aprì una nuova strada alla riscoperta dell'opera dello scrittore agrigentino.
\end{abstract}

Parole chiave: Luigi Pirandello, Adam Hanuszkiewicz, Così è se vi pare), adattamento, ricezione, nuovo paradigma drammatico 


\begin{abstract}
In this article we are talking about the staging of Pirandello's So It Is (If You Think So) who had a remarkable success on the stage of the Teatr Powszechny in Eódź in 1989. This representation was important not only for the career of the director, Adam Hanuszkiewicz, but also for the reception of Pirandello's dramatic production, of which the fortune was not until then particularly favorable. It proposed new interpretative possibilities hitherto hidden in the work of the Italian author. In fact, in addition to transgressing the stage conventions, according to some by now obsolete, the Sicilian put forward a new fragmentary and apparently unfinished dramaturgy that focuses rather on "life" than on "purely agonistic action". By exposing a new paradigm of the "drama-of-life" which stands in clear opposition to the "drama-in-life", that is "absolute drama", he gave birth to modern and contemporary dramaturgy. Adhering to Pirandello's theatrical and dramatic outlook, Hanuszkiewicz therefore tends to free the author of Henry IV from traditional interpretations that focused essentially on his system called "pirandellism" or on the alleged "existentialism". The Polish director did not hesitate to adopt the drama in modern times, without however affecting the "ideological" presuppositions of the work: Hanuszkiewicz reinterpreted it according to the contemporary and also deeply personal key, discovering its always current and universal character that could be accepted with understanding and admiration not only in Italy. The article therefore traces the fortune of the dramatic performance which, as same as The Mountain Giants, staged by Izabella Cywińska (1974), opened a new road to the rediscovery of the work of the writer from Agrigento.
\end{abstract}

Keywords: Luigi Pirandello, Adam Hanuszkiewicz, So It Is (If You Think So), adaptation, reception, new dramatic paradigm

L'opera letteraria è il dramma e la commedia concepita e scritta dal poeta: quella che si vedrà in teatro non è e non potrà essere altro che una traduzione scenica. Tanti autori e tante traduzioni, più o meno fedeli, più o meno felici; ma, come ogni traduzione, sempre e per forza inferiori all'originale ${ }^{1}$.

La ricezione della drammaturgia di Pirandello in Polonia è già stata sottoposta ad esame da vari studiosi. Basti citare a questo proposito il lavoro pionieristico in questo campo di Alicja Forysiak-Strazzanti ${ }^{2}$, nonché gli scritti di Monika Gurgul ${ }^{3}$ che esaminano la fortuna dello scrittore siciliano nei territori attraversati dalla Vistola. Nel primo caso, il teatro di Pirandello è illustrato attraverso la critica e la pratica teatrale polacca prima e dopo la Seconda

1 L. Pirandello (2006), Saggi e interventi, (a c. di F. Taviani), Milano, Mondadori, p. 1069.

2 A. Forysiak-Strazzanti (1993), Teatr Pirandella Polsce, Poznań, Wydawnictwo Naukowe UAM.

3 M. Gurgul (2016), "Incontri artistici con Pirandello nel teatro polacco degli anni '20 e '30 del XX secolo”, Romanica Cracoviensia, 3, pp. 189-196. 
guerra mondiale fino agli anni 1983, mentre nel secondo vengono descritti gli incontri artistici con Pirandello nel teatro prevalentemente cracoviano degli anni '20 e '30 del ventesimo secolo. A questo si potrebbe aggiungere un testo di Gabriele la Rosa ${ }^{4}$ sul successo dello scrittore di gran vaglia a Breslavia - città che ridivenne polacca dopo il crollo del regime nazista, nonché il testo di Cezary Bronowski sulla fortuna di Pirandello in Polonia negli anni Settanta e Ottanta del secolo scorso ${ }^{5}$. Queste indagini potrebbero essere approfondite e completate dalla rassegna delle rappresentazioni teatrali dell'autore di Come tu mi vuoi sulle scene di Łódź che per molti decenni fu la seconda metropoli polacca per popolazione. Anche se la città godeva nel primo Novecento, del prestigioso status di potente agglomerato industriale, essa non era considerata un vero centro culturale e artistico: ciò nonostante, il repertorio dei teatri dell'epoca, oltre all'intrattenimento, proponeva opere straniere - tra cui quelle dello scrittore italiano, che furono calorosamente accolte dal pubblico di questa città manifatturiera ${ }^{6}$. Anche dopo il 1945, quando Łódź ottenne una posizione di maggiore rilievo sulla mappa culturale della Polonia postbellica (basti citare i due grandi registi Leon Schiller e Kazimierz Dejmek, che vi lavorarono con successo), il nome dell'autore di Agrigento non vi era sconosciuto ${ }^{7}$.

I drammi di Pirandello, dunque, apparvero sui palcoscenici di molte città polacche, anche, pertanto, nelle sale teatrali della «terra promessa» come veniva chiamata Łódź ai tempi del Regno del Congresso ${ }^{8}$, benché meno spesso, a confronto con altri palcoscenici polacchi. È interessante dunque studiare al riguardo la ricezione dei drammi pirandelliani nei teatri di Łódź per riflettere sul ruolo che essi poterono svolgere nella storia teatrale della città. Senza dubbio tale approccio non tenderebbe a esaurire l'argomento, ma metterebbe in luce il percorso, a volte discreto della drammaturgia dell'autore italiano, e il suo impatto potenziale sulla vita culturale di questa città: centro dell'allora produzione tessile e centro geografico della Polonia del II dopoguerra.

4 G. La Rosa (2014), "Pirandello a Breslavia", Italica Wratislaviensia, nr 4, pp. 235-254.

5 C. Bronowski (2004), "La fortuna di Luigi Pirandello in Polonia negli anni Settanta e ottanta del Novecento”, Quad. PAU, n' 27-28, pp. 175-180.

6 Nel primo Novecento furono messe in scena: Sei personaggi in cerca d'autore, (Teatr Miejski, 1924), Enrico IV, (Teatr Miejski, 1926), L'uomo, la bestia e la virtù, (Teatr Kameralny, 1929).

7 Nel secondo Novecento furono messe in scena: Sei personaggi in cerca d'autore, (Teatr im. Jaracza, 1962), L'uomo, la bestia e la virtù, (Teatr im. Jaracza, 1966), Così è (se vi pare), (Teatr Powszechny, 1989).

8 Królestwo Kongresowe (in russo Ца́рство По́льское) fu uno stato vassalle sotto controllo dell'Impero russo, esistito dal 1815 fino al 1915. 
Ciononostante, al fine di aggirare le ripetizioni, abbiamo deciso di concentrarci esclusivamente sull'ultima rappresentazione di Pirandello, la messa in scena, cioè, di Così è (se vi pare) (1989), poiché essa segnò un cambiamento cruciale nell'interpretazione dell'opera dell'autore italiano in Polonia. Nel primo Novecento, il drammaturgo "fu giudicato anzitutto per la categorie del suo sistema filosofico - il pirandellismo, le cui principali tendenze abbracciarono lo scetticismo ed il relativismo"; ; nel dopoguerra, in seguito al disgelo. A Pirandello fu assegnata l'etichetta di anticipatore del "teatro esistenzialista". A differenza di queste due chiavi interpretative dominanti, ormai accuratamente esaminate, si fece strada l'idea di "considerare il teatro di Pirandello come incompiuto che richiede l'intervento del regista" ${ }^{10}$. I giganti della montagna (1973) di Izabella Cywińska, "un vero successo scenico del genere", incitò altri artisti a rileggere l'opera del Siciliano "con la chiave moderna": tra le varie letture possiamo annoverare quella di Adam Hanuszkiewicz (1924-2011), enfant terribile del teatro polacco. Il regista (ammiratore di Fellini), considerato un distruttore nonché un iconoclasta della "cultura polacca tradizionale", sconvolgeva il pubblico con i suoi spettacoli dove riproponeva "una visione contemporanea" dei grandi classici polacchi ed europei. Allestendo il dramma di Pirandello, Hanuszkiewicz non ne alterava pertanto il significato filosofico, letterario ed artistico, ma vi riscopriva la "modernità" di quel teatro che contribuì alla notorietà mondiale dello scrittore italiano. Quella rappresentazione dimostrò che Pirandello è sempre attuale e che va ricordato non solo come innovatore scenico, ma anche come padre del dramma moderno e contemporaneo.

Così è (se vi pare) - in polacco: Tak jest, jak się państwu zdaje - ebbe la sua première il 25 febbraio 1989 al Teatr Powszechny ${ }^{11}$ per la regia di Adam Hanuszkiewicz, la traduzione di Jerzy Jędrzejewicz, la scenografia di Xymena Zaniewska i Mariusz Chwedczuk. Va sottolineato che il dramma, a parte Enrico $I V$, Sei personaggi in cerca d'autore, L'uomo, bestia e virtù, ha riscontrato un vero successo presso il pubblico polacco a partire dal suo primo allestimento nel 1926 al Teatr Mały a Varsavia. L'ultima rappresentazione è avvenuta alla Scuola nazionale di teatro Ludwik Solski di Cracovia (Państwowa Wyższa Szkoła

9 A. Forysiak-Strazzanti, op. cit., p. 85.

10 Ibidem., p. 86.

11 Teatr Powszechny aprì le sue porte in maggio 1945 con la prima dei Partigiani di Vsevolod Ivanov. Agli inizi, la scena fu gestita dal Teatro dell'Armata Polacca (Teatr Wojska Polskiego), creato durante la Seconda guerra mondiale nell'Unione Sovietica, sciolto nel 1949, quando la compagnia drammatica si trasferì definitivamente a Varsavia rinata dalle ceneri. Fino ad oggi Teatr Powszechny gode di fama del teatro «popolare» che presenta un repertorio essenzialmente leggero rivolto ad un vasto pubblico. Vi è stato attuato il Centro della commedia. 
Teatralna im. Ludwika Solskiego) nel 1997, come spettacolo degli studenti dell'ultimo anno del dipartimento di recitazione.

Cosi è (se vi pare), con il sottotitolo "parabola in tre atti" ${ }^{12}$ annuncia i temi prediletti pirandelliani della verità relativa, dell'incomunicabilità e della solitudine umana. La vicenda si svolge nell'ambiente di una cittadina di provincia (Valdana) la cui tranquillità sarà scossa dall'arrivo del Signor Ponza e di sua suocera la Signora Frola, usciti miracolosamente salvi da un terribile terremoto. Si dice che l'uomo tenga segregata la moglie da sua madre, però nessuno ha mai visto la povera donna. La gente non si dà pace e cerca di capire la verità su questo trio insolito. Il signor Ponza e la signora Frola diventano presto oggetto di un vero interrogatorio stringente: ambedue si accusano reciprocamente di pazzia. La signora Frola spiega l'esagerata possessività del genero nei confronti di sua moglie. Il marito invece sostiene di abitare con la sua seconda moglie, dato che la prima (la presunta figlia della madre addolorata nonché sconsolata) è morta durante il sisma infausto. Queste rivelazioni discordanti sconcertano i cittadini mentre fanno ridere Lamberto Laudisi (il quale parla per conto di Pirandello) che svela l'assurdità di tutto questo voler investigare per voler scoprire ad ogni costo una verità oggettiva. Allora tocca alla moglie del signor Ponza, l'unica in grado di decifrare l'enigma, di presentare la sua versione davanti ai curiosi eccitati. Ma quest'ultima, poco disposta a soddisfare domande su argomenti insistenti, afferma di essere sia la seconda moglie del signor Ponza che la figlia della signora Frola. "Per me, io sono colei che mi si crede" 13 , conclude la signora provocando una grande perplessità dei pettegoli e risa di Laudisi trionfante.

Per il regista polacco l'opera, fermamente criticata a suo tempo da Antonio Gramsci nonché da Benedetto Croce, pare particolarmente attuale, poiché lo scrittore, desiderando interrogarsi, interroga anche il pubblico sul significato dell'azione, coinvolgendolo a pronunciarsi su ciò che sfilava davanti ai suoi occhi: "lo spettatore è sulla scena, come la scena è fra gli spettatori si è costretti a recitare la nostra parte, a farsi le domande che si fanno i personaggi e, insieme, a chiedersi che senso abbia farsele"14. L'entusiasmo con cui Hanuszkiewicz

12 Cf. «One of the most successful challenges to the bourgeois world of naturalist drama, Right You Are (If You Think So) is nevertheless set in the familiar drawing room of naturalist plays. But Right You Are presents a dramatic situation which is both incredible and implausible, like so many of Pirandello's plots. The drama's hyperbolic world, with its far-fetched circumstances, provides glimpses into the universal condition of man, grotesque as it is», F.A. Bassansese (1997), Understanding Luigi Pirandello, University of South Carolina Press, p. 45.

13 L. Pirandello (2000), Così è (se vi pare), Milano, Garzanti, p. 75.

14 N. Chiaromonte (1960), La situazione drammatica, Milano, Bompiani, p. 64, citato da G. Antonucci (2012), Storia del teatro italiano contemporaneo, Roma, Edizioni Studium, p. 36. 
realizza il dramma di Pirandello è dovuto senza dubbio al fatto che l'autore italiano esprimeva attraverso i suoi scritti la "crisi del dramma" (frattura tra la scena e la platea ${ }^{15}$ ) e cioè poteva essere considerato come un precursore del cosiddetto "teatro postdrammatico"16, termine coniato da Hans-Thies Lehmann. La pièce sembra di servire al regista teatrale come un pretesto per trascendere ogni interpretazione razionale della vita, conformemente all'idea del drammaturgo. Portare sulla scena il caos non significa affatto che Hanuszkiewicz si allontana in extremis dal presunto "logocentrismo" tanto biasimato dagli sperimentalisti degli ultimi decenni del XX secolo. Anzi, egli rivendica, come già tanti altri in precedenza, il diritto a riscrivere a suo modo un testo originale, enfatizzando "l'autonomia totale della testualità spettacolare nei confronti di quella letteraria di partenza" ${ }^{17}$. Su questo punto il regista si sarebbe ispirato alle parole stesse di Pirandello espresse dal dottor Hinkfuss in Questa sera si recita a soggetto: "in teatro l'opera dello scrittore non c'è più"18. E poi, dilungandosi sulla singolarità (e indiscutibilmente sulla superiorità) della regia rispetto al testo teatrale, questi non manca di precisare:

Se un'opera d'arte sopravvive è solo perché noi registi possiamo ancora rimuoverla dalla fissità della sua forma; sciogliere questa sua forma dentro di noi in movimento vitale; e la vita gliela diamo allora noi; di tempo in tempo diversa, e varia dall'uno all'altro di noi; tante vite, e non una; come si può desumere dalle continue discussioni che se ne fanno e che nascono dal non voler credere appunto questo: che siamo noi a dar questa vita, sicché quella che do io non è affatto possibile che sia uguale a quella di un altro ${ }^{19}$.

La rappresentazione del dramma rende conto delle preoccupazioni dell'autore siciliano. Jerzy Kwieciński dedica alcune osservazioni sommarie allo spettacolo che giudica un successo anche se il teatro di Pirandello sembra, secondo il critico teatrale, non destare più l'attenzione del pubblico come nel primo dopo guerra ${ }^{20}$. Il recensore, non esitando a notare dei momenti noiosi della rappresentazione (in particolare scene eccessivamente lunghe della

15 «Anche Pirandello era persuaso che teatro e dramma fossero incompatibili», H.-T. Lehmann (2017), Il teatro postdrammlatico, trad. S. Antinori, Cue Press, p. 54.

16 A. Petrini (2018), "Il teatro postdrammatico e i suoi ampi dintorni", Mimesis Journal, 7, 1, pp. 39-75.

17 M. Pieri (2017), “Teatro e letteratura”, [in:] L. Allegri (a c. di), Il teatro e le arti. Un confronto fra linguaggi, Roma, Carocci, p. 24.

18 L. Pirandello (2007), Questa sera si recita a soggetto, in: Maschere nude, Roma, Newton Compton editori, p. 110.

19 Ibidem., p. 111.

20 J. Kwieciński, Igraszki z rzeczywistościa, „Odgłosy”, 19/03/1989. 
prima parte), apprezza invece il finale di Hanuszkiewicz, durante il quale, contrariamente al testo originale, continua lo spettacolo per dare spazio all'espressione di natura ontologica. Gli piace pure l'idea del regista di aver fatto dei frequentatori di casa di Agazzi dei fantocci senza vita, come se fossero azionati per mezzo di fili. Queste marionette sembrano simboleggiare delle figure di atrofia linguistica: loro parlano, ma non dicono niente, segno pessimista dell'epoca del regno del "grande mutismo" ${ }^{21}$ in cui, paradossalmente, tutti mettono in giro voci fatte di clichés sprovvisti di ogni significato. Concludendo il suo pezzo, Kwieciński non manca di rivolgere dei complimenti agli attori: tra tutti si distinguono i personaggi dell'anziana signora Frola (Jadwiga Siennicka), del signor Ponzo (il tremante Robert Rozmus) e soprattutto la misteriosa donna velata (un'eccellente interpretazione di Magdalena Cwenówna).

Anche Henryk Pawlak ammira la scena finale (ai suoi occhi probabilmente il momento più intrigante dello spettacolo ${ }^{22}$ ) che, secondo lui, sottolinea l'attualità del testo pirandelliano, specie a proposito dei presupposti filosofici del drammaturgo. Giudicando superate le proposte delle riforme teatrali pirandelliane alle soglie del terzo millennio, l'autore della recensione osserva che il Hanuszkiewicz tenta di rileggere l'opera di Pirandello adattandola per lo spettatore contemporaneo. $\mathrm{Al}$ regista polacco piace la dimensione paradossale e tragicomica del dramma che mette in dubbio non soltanto le convenzioni sceniche, ma anche i condizionamenti sociali assurdi. Di fatti, tutto si svolge in una atmosfera grottesca, i personaggi si muovono come le marionette dimostrando in tal modo l'assurdità della loro condizione precaria, e cioè, quella di un'esistenza umana traballante. Hanuszkiewicz avrebbe inteso fare del palcoscenico, come Jean Genet ${ }^{23}$, uno specchio in cui il pubblico poteva guardarsi. L'artificialità dei gesti goffi ed innaturali di questi "pupazzi umani", potevano suscitare l'ilarità degli spettatori, ma nello stesso tempo, risvegliare in loro una riflessione esistenziale. Tutto avviene come se il regista volesse illustrare drammaticamente il concetto di umorismo ${ }^{24}$ di Pirandello, mettendo in primis l'avvertimento del contrario per poi passare al sentimento del contrario. Pawlak sostiene che, anche se Hanuszkiewicz non ha osservato fedelmente la struttura drammatica dell'opera, egli rimane sempre devoto al suo contenuto attinente al pensiero pessimista dello scrittore italiano. Il regista desidera quindi

21 Cfr. W. Horzyca (1969), Poeta żywej maski, [in:] O dramacie, Warszawa, p. 190, citato da A. Forysiak-Strazzanti, op. cit., p. 44.

22 H. Pawlak, Pani Frola i inni, „Głos robotniczy”, 3/03/1989.

23 Cfr. D. Bradby (1984), Modern French Drama 1940-1980, Cambridge University Press, p. 178.

24 L. Pirandello (2009), L'umorismo, (a c. di) Maria Argenziano, Roma, Newton Compton, p. 148. 
che il pubblico prenda parte allo spettacolo, fa entrare gli attori dalla platea, abolendo così la quarta parete. Lo spettatore partecipa dunque al divertimento (prima parte) che verso la fine (seconda parte) si trasforma in un colloquio fra i personaggi e l'Autore, un dialogo sulle questioni essenziali della vita a cui viene invitato anche il pubblico. Tutti i critici mettono l'accento su questo contrasto, tra il comico e il tragico, capace di destabilizzare lo stato d'animo dell'audience.

Lucyna Skompska ${ }^{25}$, che dà un resoconto più dettagliato nonché più ampio della rappresentazione, percepisce Pirandello come un incontestabile innovatore teatrale, però estraneo alle ricerche formali di Edward Gordon Craig, Alexandr Tairov o Vsevolod Meyerhold che condussero loro al rifiuto del testo drammatico a favore dell'espressione prettamente spettacolosa (consono alla celebre parola d'ordine: "riteatralizzare il teatro"). Anche l'autore italiano si vuole distruttore delle convenzioni teatrali obsolete, ma attraverso il dialogo, poiché rimane sempre attaccato alla dimensione letteraria del dramma senza che lui dimentichi, come lo definisce Jean-Pierre Sarrazac, il suo "devenir scénique"26. Come sottolinea Skompska, il drammaturgo rigetta il realismo (paradossalmente salvaguardando la sua poetica) per dimostrare la falsità del teatro, i meccanismi ed i principi all'origine dello spettacolo che svelano allo stesso tempo la complessità della vita e della personalità umana. È proprio per questo motivo che Pirandello, prosegue Skompska, esige che gli attori si immedesimino con i personaggi (imbeversi), che la loro interpretazione sia impulsiva ed irruenta come nella commedia dellarte senza tuttavia osservare alcun elemento convenzionale e polveroso. Hanuszkiewicz sembra ottemperare alla doxa pirandelliana, poiché i personaggi entrano in scena chiassosi e molto espressivi. Si direbbe che gli attori recitino con una certa spontaneità conformemente alle prescrizioni comiche tradizionali. Eppure, la loro "naturalezza" è artificiale perché calcolata dal regista, che rende i personaggi delle marionette che gridano e si comportano senza una logica apparente. E questo, secondo Skompska, non corrispondeva affatto alle intenzioni del drammaturgo italiano. Anche la scenografia, così dettagliata, non rispecchiava le didascalie dell'autore e Lamberto Laudisi, portavoce di Pirandello, invece di essere un saggio scettico sulla verità assoluta come viene descritto nel dramma, diventa nello spettacolo un buffone, mentre la signora Frola, una nobile vecchietta, si atteggia a una civetta avanti negli anni.

Lo spettacolo cambia nel momento in cui appare la signora Ponza, è il punto di svolta dell'azione. Questa arriva dalla platea e non come gli altri attori, da dietro le quinte, come se il regista volesse sottolineare che la donna entrava

25 L. Skompska, Pirandello na tódzkiej scenie, „Teatr”, 01/06/1989.

26 J.-P. Sarrazac (2012), Poétique du drame moderne. De Henrik Ibsen à BernardMarie Koltès, Paris, Éditions du Seuil, p. 11. 
in scena da parte della vita. Anche l'Autore (un altro personaggio immaginato da Hanuszkiewicz) giunge nel palcoscenico dalla parte degli spettatori. L'uomo si adagia nella poltrona per leggere un giornale, assolutamente insensibile a tutto ciò che lo circonda. Allo stesso tempo, le ultime battute della Ponza si trasformano bruscamente in un monologo drammatico tratto da Sei personaggi in cerca d'autore, monologo rivolto ad Autore che sembra sempre noncurante, immerso nella lettura della sua rivista. L'indifferenza di questi è messa in evidenza, dato che il passaggio citato dall'opera maggiore di Pirandello toccava proprio il tema dell'incomunicabilità interumana. Per di più, Hanuszkiewicz sceglie certe battute dell'uomo morente di L'uomo dal fiore in bocca, perché, proprio in quelle l'autore italiano intendeva rivolgersi, "nel modo più semplice e nitido, al di là di troppo complessi giochi teatrali, verso una contemplazione del fondo oscuro di sofferenza della condizione umana"27. A quel punto gli attori rimangono sbalorditi non sapendo cosa fare in questa situazione insolita, una certa confusione mette anche il pubblico in imbarazzo che, perplesso al pari dei commedianti, non sa se lo spettacolo sia giunto alla sua fine. La tela non si abbassa poiché non c’è. Eppure, ha inizio un dialogo sconcertante che comincia la signora Ponza. Questa fa pensare a un fantasma, a un simbolo incomprensibile di un'idea umana, che non si inquadra in nessuna convenzione teatrale. Gli attori si rendono conto che anche loro sono diventati spettatori e spariscono dietro le quinte mentre la signora Ponza tenta di suicidarsi. Si allontana. Si sente prima uno sparo e subito dopo la sirena di un'autoambulanza. Sul palcoscenico irrompono infermieri indaffarati con una barella, ma la signora Ponza vive, anzi sta bene. Lei non può morire, poiché, come nota senza malizia Skompska, lei è eterna al pari delle "idee platoniche" 28 . Hanuszkiewicz - e ancora il pensiero della recensora - presenta il conflitto, come ha segnalato José Ortega y Gasset nella sua Disumanizzazione dell'arte, tra le idee non quello tra i personaggi, a cui ci ha abituato il dramma classico. Il destino di sei personaggi, di cui parla il filosofo e saggista spagnolo, sarebbe unicamente un pretesto per mettere avanti un dramma di certi concetti soggettivi, fantasmi nati nell'immaginazione dell'autore italiano. Questa opinione è condivisa da Ewa Łubieniewska, secondo la quale, nello spettacolo di Hanusziewicz, lo stereotipo è stato sostituito dal personaggio (in questo caso la signora Ponza) che, tentando di togliersi la vita, cercava di sentire disperatamente la realtà della sua esistenza ${ }^{29}$. Alla fine dello spettacolo (questa volta decisiva) gli operai del teatro si mettono a smontare lo

27 G. Ferroni (2017), Storia della letteratura italiana. Il Novecento e il nuovo millennio, Milano, Mondadori Università, p. 146.

28 L. Skompska, op. cit.

29 E. Eubieniewska (2017), Telewizyjne gry $z$ Pirandellem, "Annales Universitatis Paedagogicae Cracoviensis”, Studia Historicolitteraria 17, p. 146. 
scenario lasciando intravedere sullo sfondo tutta la macchina teatrale. In più, si sentono voci dei buttafuori che tramite i megafoni danno ordini di sgomberare il palcoscenico. Allora, come precisa Skompska, le finalità scenografiche del regista si fanno più intellegibili: egli voleva dimostrare l'artificialità della convenzione comica del primo tempo dello spettacolo che sfocia, alle fine, in un dramma esistenziale. Skompska ritiene che Hanuszkiewicz illustri, attraverso la sua rappresentazione, i tre strati dellazione drammatica di Pirandello: "dove si svolge un vero dramma? - si chiede a questo proposito - sul palcoscenico, dove la convenzione entra in conflitto con la vita? oppure nella coscienza dei personaggi che chiedono una piena vita? o forse unicamente nella coscienza dell'autore?"30

Concludendo il suo pezzo, Skompska non può che deplorare la mancanza del riso filosofico di Laudisi, quello che si prende gioco di ogni tentativo di cercare un'unica ed immutabile verità. Forse quel riso sarebbe, agli occhi di Skompska, il vero protagonista del dramma come accade in All'uscita del teatro dopo la rappresentazione di una nuova commedia di Nikolaj Gogol' (1842), dove alla domanda sul come mai, nel Revisore, non ci sia nemmeno un protagonista simpatico, un personaggio del dramma risponde: "Ma sì! Quel personaggio è proprio la risata!"31

Nelle sue conclusioni, Alicja Forysiak-Strazzanti auspica la ricerca di testi pirandelliani sconosciuti, "affinché essi possano costituire la base di un lavoro autonomo dei realizzatori" ${ }^{2}$. Non ricusando questo suggerimento, potremmo dire che vale la pena di rivisitare la drammaturgia (anche quella "classica") di Pirandello, che non è solamente la dimostrazione dell'acrobazia intellettuale, come la commedia dell'arte era la dimostrazione dell'acrobazia fisica ${ }^{33}$, ma è la scrittura con cui egli intende rompere i precetti, spezzare i vincoli delle norme aristoteliche. Essa segna un passo verso la "letteratura teatrale" moderna, che schiva le strette regole del dramma tradizionale. A questo proposito, alcuni critici sottolineano a ragione l'importanza di Pirandello nello sviluppo della drammaturgia mondiale inclusa quella polacca ${ }^{34}$. Senza

30 Ibidem.

31 L. Skompska, op. cit.

32 A. Forysiak-Strazzanti, op. cit., p. 86.

33 M. Brahmer nell'introduzione al libro: L. Pirandello (1960), Dramaty, Warszawa, Państwowy Instytut Wydawniczy, p. 21.

34 Józef Kelera confronta l'opera di Pirandello con Witkacy, Witold Gombrowicz, Tadeusz Różewicz, Jerzy Grotowski (J. Kelera, Pirandello widziany z Polski, „Odra” 1987), mentre Sylwia Kucharuk paragona la drammaturgia dell'autore italiano con 
dubbio l'opera pirandelliana non è ancora ben conosciuta lungo la Vistola, ma la rappresentazione di Hanuszkiewicz si iscrive in quel tentativo di riscoprire le potenzialità estetiche finora occultate del teatro dell'intellettuale agrigentino. Secondo Alonge, Pirandello è forse il più futurista degli autori italiani di quel primo Novecento a far "straripare il dramma fuori del palcoscenico senza limiti di tempo e di spazio"35. Sotto questo aspetto, Kazimierz Braun ${ }^{36}$ colloca Pirandello tra gli autori della Grande Riforma del teatro, poiché è riuscito a trasgredire le convenzioni sceniche ${ }^{37}$ (teatro senza netta divisione fra scena e aera per gli spettatori). Eppure, l'opera di Pirandello, saltando l'ideale della barriera che esiste tra palco e pubblico non abolisce esclusivamente la quarta parete, ma anche mette avanti una nuova drammaturgia, d'ora in poi, frammentaria, slegata, incompiuta, incentrata sulla "vita" e non sull'"azione" ${ }^{2}$. Ed è proprio questa la chiave con cui Hanuszkiewicz legge il dramma del Siciliano che propone un nuovo paradigma del «dramma-della-vita» che sta in nitida opposizione al «dramma-nella-vita», ossia «dramma assoluto» (Peter Szondi). Hanuszkiewicz riscrive il teatro di Pirandello senza però ledere, intaccare i suoi presupposti filosofici e artistici. Egli sembra capire la "distruzione voluta" (da parte stessa dell'autore) della forma drammatica, che apre una nuova via allo sviluppo della drammaturgia moderna e contemporanea ${ }^{39}$. Essa non significa una crisi oppure un superamento del dramma stesso (ancora meno la sua morte, diagnosi promulgata con successo da Adorno e Lehmann), ma piuttosto la sua dissolutezza che si affranca dalla tirannide delle costrizioni aristoteliche. Non nega la dimensione letteraria della drammaturgia (anche meno la sua potenzialità scenica), ma i suoi dettami diventati obsoleti, e cioè, non corrispondenti alle aspettative del pubblico odierno. Hanuszkiewicz ci

quella di Jerzy Szaniawski (S. Kucharuk, Pirandello i Szaniawski: przyczynek do badań komparatystycznych, Wydawnictwo UMCS, Lublin, 2011), le stesse analogie sono accentuate da Cezary Bronowski nel suo libro: Historia dramaturgii wtoskiej XX wieku, (Wydawnictwo Uniwersytetu Wrocławskiego, 2008, pp. 80-85). Per saperne di più consultare: S. Kucharuk (2012) Polska krytyka wobec dramatów Pirandella, „Italica Wratislaviensia”, nr 3, pp. 107-121.

35 Citato da F. Perrelli (2016), "Il teatro italiano del primo Novecento", [in:] F. Perrelli (a c. di) Storia europea del teatro italiano, Roma, Carocci editore, p. 318.

36 Egli stesso metterà in scena I giganti della montagna a Danzica nel 1962.

37 K. Braun (1984), Wielka Reforma Teatru w Europie, Ossolineum, p. 291.

38 "Proprio questa irruzione della vita che violenta la struttura chiusa della scena tradizionale; le sue opere non sono esemplari di puro sperimentalismo teatrale, anche se esaltano in un crogiolo di procedimenti scenici inusitati le motivazioni profonde dell'avanguardia novecentesca", N. Borsellino, [in:] L. Pirandello, Così è (se vi pare), op.cit., p. 31.

39 J.-P. Sarrazac, op. cit., pp. 11-29. 
invita dunque a rileggere la drammaturgia di Pirandello da una prospettiva contemporanea che conferisce tanto spazio creativo a un regista d'oggi.

\section{Bibliografia}

Antonucci, Giovanni (2012). Storia del teatro italiano contemporaneo, Roma, Edizioni Studium. Bassansese, Fiora A. (1997). Understanding Luigi Pirandello, University of South Carolina Press. Bradby, David (1984). Modern French Drama 1940-1980, Cambridge University Press.

Braun, Kazimierz (1984). Wielka Reforma Teatru w Europie, Warszawa, Ossolineum.

Bronowski, Cezary (2004). La fortuna di Luigi Pirandello in Polonia negli anni Settanta e ottanta del novecento, „PAU”, nr 27-28, pp. 175-180.

Ferroni, Giulio (2017). Storia della letteratura italiana. Il Novecento e il nuovo millennio, Milano, Mondadori Università.

Forysiak-Strazzanti, Alicja (1993). Teatr Pirandella w Polsce, Poznań, Wydawnictwo Naukowe UAM.

Gurgul, Monika (2016). Incontri artistici con Pirandello nel teatro polacco degli anni '20 e '30 del XX secolo, Romanica Crnacoviensia, 3, pp. 189-196.

La Rosa, Gabriele (2014). Pirandello a Breslavia, Italica Wratislaviensia, nr 4, pp. 235-254.

Lehmann, Hans-Thies (2017). Il teatro postdrammatico, trad. Sonia Antinori, Cue Press.

Łubieniewska, Ewa (2017). “Telewizyjne gry z Pirandellem”, Annales Universitatis Paedagogicae Cracoviensis, Studia Historicolitteraria, pp. 135-149.

Perrelli, Franco (2016). "Il teatro italiano del primo Novecento", [in:] F. Perrelli (a c. di) Storia europea del teatro italiano, Roma, Carocci editore, pp. 193-320.

Petrini, Alfio (2018). "Il teatro postdrammatico e i suoi ampi dintorni", Mimesis Journal, 7. pp. 39-75.

Pieri, Marzia (2017). “Teatro e letteratura”, [in:] Luigi Allegri (a c. di), Il teatro e le arti. Un confronto fra linguaggi, Roma, Carocci.

Pirandello, Luigi (1960). Dramaty, (a c. di) M. Brahmer, Warszawa, Państwowy Instytut Wydawniczy.

Pirandello, Luigi (2000). Così è (se vi pare), Milano, Garzanti.

Pirandello, Luigi (2006). Saggi e interventi, (a c. di) F. Taviani, Milano, Mondadori.

Pirandello, Luigi (2007). Questa sera si recita a soggetto, [in:] Maschere nude, Roma, Newton Compton editori.

Pirandello, Luigi (2009). L'umorismo, (a c. di) Maria Argenziano, Roma, Newton Compton editori.

Sarrazac, Jean-Pierre (2012). Poétique du drame moderne. De Henrik Ibsen à Bernard-Marie Koltès, Paris, Éditions du Seuil.

\section{Sitografia}

Kwieciński, Jerzy (19/3/1989). „Igraszki z rzeczywistością”, Odgłosy, http://www.e-teatr.pl/ $\mathrm{pl} /$ realizacje/107, autor.html [11/10/2019].

Pawlak, Henryk (3/03/1989). „Pani Frola i inni”, Głos robotniczy, http://www.e-teatr.pl/pl/ realizacje/107, autor.html [03/12/2019].

Skompska, Lucyna (01/06/1989). „Pirandello na tódzkiej scenie”, Teatr, http://www.e-teatr.pl/ $\mathrm{pl} /$ realizacje/107,autor.html [15/10/2019]. 